\title{
The importance of contextual relevance
}

To the Editor - Our original experiment was designed to see whether participants thought of the Internet as a transactive memory source ${ }^{1}$. The question we hoped to answer was: When people don't know the answer to something, will they think of the Internet? To answer this question, we ran two blocks of a modified Stroop task: each Stroop word was seen only once by participants, and before each word, they were shown a different six digit number, which was reported back by them after each Stroop word. We found that target words about the Internet had longer reaction times compared with non-target words, particularly after the hard question block.

The procedure described in the linked replication study ${ }^{2}$ indicates that Stroop words were repeated four times and that one six digit number was given to participants to memorize across the entire block of trials. This is the procedure for active thought suppression ${ }^{3}$ : when people are asked explicitly not to think about a single target word, they must engage in active suppression. In such cases, one would expect post-suppression rebound, which would mean that there would be increased reaction times for repeated trials of the same target word. However, in both our original experiment ${ }^{1}$ and the replication ${ }^{2}$, participants were not asked to suppress a single target word about computers; for this word, we would not expect a postsuppression rebound, which is what the data for both the original and replication study show: there was no difference in reaction time between these target and non-target words. However, the point of our original study ${ }^{1}$ was to understand whether people thought of the Internet when they wanted to know something, not whether they suppressed thoughts of the Internet when they were asked to do so.

When looking at the target words we used in the experiment, I was struck by how specific they were to the time the experiment was run. We originally ran this experiment in 2006, one year before the first iPhone was released. Accessing the Internet at this time required one to sit at a computer, with keyboard, mouse and ethernet cable, navigate to a browser on the desktop, and type a query. As this was the only way to access the Internet, it was easier to come up with a list of words that everybody would have similar experience with. We included: Google, Yahoo, mouse, keys, Internet, browser, computer, screen, Altavista, Wikipedia, disk, Lycos, Netscape, modem, router, online. In the 12 years since we ran this experiment, however, the experience of accessing the Internet has changed dramatically. While one can still access the Internet from a desktop, it is no longer necessary: people use their phones, type or use voice command, and usually own multiple different devices that require different procedures for going online. Therefore, the specific target words that we used are somewhat obsolete, and were likely even dated by the time the paper was published (2011). If I were attempting to replicate our original study ${ }^{1}$ today, I would focus primarily on target words about phones (as they seem most ubiquitous), but would also be sure to pre-test many possible words to ensure their contextual relevance before putting them into the modified Stroop.

\section{Betsy Sparrow Shrewsbury, MA, USA. \\ e-mail: betsysparrow@gmail.com}

Published online: 27 August 2018

https://doi.org/10.1038/s41562-018-0411-7

\section{References}

1. Sparrow, B., Liu, J. \& Wegner, D. M. Science 333, 776-778 (2011)

2. Camerer, C. F. et al. Nat. Hum. Behav. https://doi.org/10.1038/ s41562-018-0399-z (2018).

3. Wegner, D. M. \& Erber, R. J. Pers. Soc. Psychol. 63, 903-912 (1992).

Competing interests

The author declares no competing interests. 